\title{
Primate Motor Cortex and Free Arm Movements to Visual Targets in Three-Dimensional Space. II. Coding of the Direction of Movement by a Neuronal Population
}

\author{
Apostolos P. Georgopoulos, Ronald E. Kettner, ${ }^{a}$ and Andrew B. Schwartz ${ }^{\mathrm{b}}$ \\ The Philip Bard Laboratories of Neurophysiology, Department of Neuroscience, The Johns Hopkins University, School of \\ Medicine, Baltimore, Maryland 21205
}

\begin{abstract}
We describe a code by which a population of motor cortical neurons could determine uniquely the direction of reaching movements in three-dimensional space. The population consisted of $\mathbf{4 7 5}$ directionally tuned cells whose functional properties are described in the preceding paper (Schwartz et al., 1988). Each cell discharged at the highest rate with movements in its "preferred direction" and at progressively lower rates with movements in directions away from the preferred one. The neuronal population code assumes that for a particular movement direction each cell makes a vectorial contribution ("votes") with direction in the cell's preferred direction and magnitude proportional to the change in the cell's discharge rate associated with the particular direction of movement. The vector sum of these contributions is the outcome of the population code (the "neuronal population vector') and points in the direction of movement in space well before the movement begins.
\end{abstract}

Evidence was presented in the preceding paper (Schwartz et al., 1988) that individual neurons in the motor cortex of the monkey are broadly tuned with respect to the direction of arm movements toward visual targets in three-dimensional (3-D) space. This suggests that, although single cells possess a preferred direction, many cells will be active for any particular movement and thus that the generation of movement in a particular direction depends upon the activity in the neuronal ensemble. We tested this "population coding" hypothesis by assuming that contributions from individual neurons add vectorially to yield a neuronal population vector. We found that this population vector accurately predicted the direction of movement in space before the onset of movement. These findings generalize to 3-D space previous results obtained in two-dimensional (2-D) space

Received Aug. 3, 1987; revised Dec. 10, 1987; accepted Dec. 10, 1987.

This work was supported by United States Public Health Service Grant NS17413, which we gratefully acknowledge. We thank Dr. Scott Zeger, Department of Biostatistics, The Johns Hopkins University, School of Hygiene and Public Health, for statistical advice. The color plots in this paper were produced using the Interactive Graphics Facility of the Department of Biophysics, The Johns Hopkins University, School of Medicine. This facility was established and maintained by NIH and NSF grants and by a gift from the Richard-King Mellon Foundation. R.E.K. was a research associate, 1985-1987. A.B.S. was a postdoctoral fellow, 1984-1987.

Correspondence should be addressed to Apostolos P. Georgopoulos, Bard Laboratories of Neurophysiology, Department of Neuroscience, The Johns Hopkins University, School of Medicine, 725 North Wolfe Street, Baltimore, MD 21205.

"Present address: Department of Psychology, Psychology Building, Indiana University, Bloomington, IN 4/405.

"Present address: Division of Neurobiology, St. Joseph's Hospital and Medical Center, Barrow Neurological Institute, 350 West Thomas Road, Phoenix, AZ 85013.

Copyright (C) 1988 Society for Neuroscience $0270-6474 / 88 / 082928-10 \$ 02.00 / 0$
(Georgopoulos et al., 1983, 1984). The population vector is a robust predictor of the direction of movement in space and is relatively immune to cell loss. Preliminary findings were published elsewhere (Georgopoulos et al., 1986; Schwartz et al., 1986).

\section{Materials and Methods}

Neuronal population. The neuronal population used in the present analyses consisted of the 475 directionally tuned cells studied by Schwartz et al. (1988). The electrical signs of their activity were recorded extracellularly in the motor cortex of 2 rhesus monkeys while the animals reached towards visual targets in 3-D space. The movements started from a central point located at shoulder level in front of the animal and in the midsagittal plane; they were of equal amplitude and of 8 different directions (see Fig. 1 in the preceding paper, Schwartz et al., 1988). The movements were monitored as described in Schwartz et al. (1988). The directional tuning function and conventions for the vector notation adopted in this paper are described in Appendix 1.

Population coding. We sought a prediction of movement direction, $\mathbf{M}$, from the neuronal population. For that purpose the following were assumed. First, the $i$ th neuron makes a vectorial contribution ("votes") in its preferred direction, $\mathbf{C}_{i}$, with a magnitude given by a weighting function, $w_{i}(\mathbf{M})$, described in the next section. And second, these contributions sum vectorially to yield the outcome of the ensemble operation, the neuronal population vector, $\mathbf{P}(\mathbf{M})$ :

$$
\mathbf{P}(\mathbf{M})=\sum_{i=1}^{1} w_{i}(\mathbf{M}) \mathbf{C}_{i}
$$

where $N$ is the number of cells in the population. The hypothesis to be tested is that the direction of the population vector is congruent with the direction of the movement. We evaluated the outcome of the population analysis by calculating 2 measures: the spherical correlation, $p$ (Fisher and Lee, 1986), between the population vector and the movement, and the angle between these 2 vectors.

Weighting function. The preferred direction, $\mathbf{C}_{i}$, in which the vectorial contribution of the $i$ th cell is made in the population, is the same for different movements. However, the magnitude, $w_{i}(\mathbf{M})$, of this contribution depends on the discharge rate of the neuron during movement in direction M. Various weighting functions were tested; they are described in detail in Appendix 2. They included functions based on experimentally observed discharge rates (weighting functions $1-6$ in Appendix 2) and others (weighting functions 7-12 in Appendix 2) based on discharge rates predicted by the directional tuning equation (Eq. 11 in Appendix 1). Two of the weighting functions were based on unnormalized discharge rates (weighting functions 1 and 7 in Appendix 2), whereas the remainder were based on rates normalizcd with respect to various parameters (weighting functions 2-6 and 8-12 in Appendix 2).

Directional variability of the population vector. The directional variability of the population vector was estimated using statistical bootstrapping techniques (Diaconis and Efron, 1983). These methods estimate the variability of a parameter by calculating the parameter a large number of times from random samples of the original sample. The variability of the parameter can then be obtained directly. In addition to this 
"statistical" variability, we estimated the variability in the direction of the population vector due to variation in cell discharge from trial to trial. This latter variability amounts to variation in the length of the constituent vectors participating in the population operation. Since there were several repetitions of each movement in the experiment, an estimate of the trial-to-trial variability in cell discharge could be obtained. We then assumed that the discharge rate, $d_{i}(\mathbf{M})$, of the $i$ th cell for a certain movement in direction $\mathbf{M}$ was distributed normally across trials with mean $d(\mathbf{M})$ and variance the experimentally observed from trialto-trial for that cell; particular values of cell discharge for a simulated trial were selected randomly from that distribution.

Three analyses were performed for estimating the directional variability of the population vector (see Table 1). The first involved repeated random sampling of the experimentally observed population $(N=475)$ without incorporating the trial-to-trial variability in cell discharge; the mean discharge rate, $d(\mathbf{M})$, was used instead. In the second analysis the original 475 cells were used, but their activity was made to vary according to their trial-to-trial variability estimated experimentally and applied in this analysis as described above. Finally, the third analysis incorporated both the random sampling procedure employed in the first analysis and the trial-to-trial variability employed in the second analysis above.

The general procedure used for these analyses was as follows. Consider a particular direction of movement. (1) First, 100 populations were created, each of size $N=475$, from the original, experimentally observed population. These new populations differed in composition (i.e., which cells were included) from each other in the first and third analyses, since they were generated by sampling randomly with replacement from the original population $(N=475)$; we mean by "random sampling with replacement" that constituent cells were selected randomly from the original population and that a particular cell that happened to be selected once was still eligible for selection for as many times as the random sampling procedure would choose it. For the second analysis, the population consisted only of the original 475 cells. (The trial-to-trial variability was incorporated in the discharge rate for the second and third analyses, but not for the first analysis.) (2) One hundred population vectors were calculated from these 100 populations using weighting function 8 (see Appendix 2) and normalized to unit length. This weighting function was based on discharge rates predicted by the directional tuning function and, therefore, can be applied to directions other than those studied experimentally in the present experiments. (3) The mean direction of the 100 population vectors was computed and the angle between this mean population vector and each of the 100 contributing vectors calculated. (4) The resulting 100 angles were rank-ordered. The 95 th percentile was used as the half-angle, $\delta$, at the apex of a $95 \%$ confidence conc for the direction of the population vector.

Effect of population size on the variability of the direction of the population vector. The effect of the population size (i.e., the number of cells in the population) on the directional variability of the population vector was studied by calculating this vector from populations of various sizes, $N$, and computing the corresponding angle $\delta$ as described for the third analysis in the preceding section. Twenty population sizes were used with $N$ varying from 10 to 475 cells (see Results) for each of the 8 movement directions tested. For a population of size $N, 100$ samples of $N$ cells were obtained from the parent population of 475 cells using random sampling with replacement. Thus, 100 subpopulations were obtained for each combination of population size and movement direction, and 100 population vectors calculated. The mean $\delta$ for each population size and direction, and the grand mean $\delta$ for a given population size across all movement directions were computcd. The latter served as an estimate of the effect of population size on the directional variability of the population vector.

Evolution of the population vector in time. We determined the population vector every $20 \mathrm{msec}$ during the reaction and movement time for each of the 8 movement directions used in the task as follows. Eight time histograms, one for each movement direction, were constructed for each of the 475 cells in the population using a $20 \mathrm{msec}$ binwidth. The histograms were aligned to the onset of the movement. A time series of population vectors was then created by calculating the population vector every $20 \mathrm{msec}$ using the following weight for the contribution of the $i$ th cell:

$$
w_{i}(\mathbf{M}, t)=d_{i}(\mathbf{M}, t)-a_{i}
$$

where $w(\mathbf{M}, t)$ is the magnitude of the contribution of the $i$ th cell's vector to the population vector at time bin $t$ for movement $\mathbf{M}$ (the value
Table 1. Sources of variability included in estimating the directional variability of the population vector (see text for details)

\begin{tabular}{lll} 
Analysis & $\begin{array}{l}\text { Random sampling of } \\
\text { the original population }\end{array}$ & $\begin{array}{l}\text { Trial-to-trial } \\
\text { variability in } \\
\text { discharge }\end{array}$ \\
\hline First & Yes & No \\
Second & No & Yes \\
Third & Yes & Yes \\
\hline
\end{tabular}

of $t$ will be negative or positive, corresponding to a time bin preceding or following the onset of movement, respectively); $d_{i}(\mathbf{M}, t)$ is the average (over 5-8 repetitions) frequency of discharge of the $i$ th cell at time bin $t$, and $a$, is the average (over 5-8 repetitions) frequency of discharge of the cell during the last $0.5 \mathrm{sec}$ of the control time. The population vector, $\mathbf{P}(\mathbf{M}, t)$, for successive time bins $t$ is

$$
\mathbf{P}(\mathbf{M}, t)=\sum_{i=1}^{x} w_{i}(\mathbf{M}, t) \mathbf{C}_{i}
$$

where $\mathbf{C}_{i}$ is the preferred direction of the $i$ th cell.

Construction of "neural" trajectories. The time series of population vectors in Equation 3 above is based on the changing patterns of activity of motor cortical neurons. These patterns, and the resulting population vectors, can be considered to represent in the motor cortex the spatial plan of the movement before the movement begins. We visualized this plan by assuming that the instantaneous direction and length of the population vector is reflected, following a time delay, in the instantaneous direction and length of the upcoming movement trajectory. For this analysis we generated a time series of population vectors using histograms of neuronal discharge aligned to the onset of movement as described above.

Let $t$ be the time bin relative to the onset of movement. We represent an actual movement trajectory in 3-D space as a consecutive series of movements, each of $20 \mathrm{msec}$ duration; the trajectory can then be visualized as a spatial series of vectors of length $s(t)$ and direction $\mathbf{M}(t)$, where $\mathbf{M}$ is a vector of unit length, connected tip to tail as $t$ increases:

$$
\{s(t) \mathbf{M}(t)\}, \quad t=1, \ldots, n .
$$

For the purpose of comparison of the actual (see Schwartz et al., 1988) with the "neural" trajectories, the length of the ongoing movement vectors was normalized with respect to the maximum vector length, $s_{\max }$, in the series $(t=1, \ldots, n)$ :

$$
\left.\left\{s^{\prime}(t)\right\}=s(t) / s_{\max }\right\}, \quad t=1, \ldots, n .
$$

Therefore,

$$
\left\{s^{\prime}(t) \mathbf{M}(t)\right\}, \quad t=1, \ldots, n .
$$

The normalized trajectory (expression 6 ) was plotted as follows. The first vector $(t=1)$ started from the point of origin of the movement, and subsequent vectors $(t=2,3, \ldots, n)$ were attached to each other tip to tail.

A neural representation of the movement trajectory based only on directional information from the population vector was constructed as follows. Let $\tau$ be the time delay between the first change in the population vector and the onset of movement, $\mathbf{P}(t-\tau)$ be the population vector at time $t-\tau$, and let $\mathbf{Q}(t-\tau)$ be a vector of unit length in the direction of $\mathbf{P}(t-\tau)$. Then, the normalized trajectory predicted from the time varying direction of the population vector and the length of the movement is

$$
\left\{s^{\prime}(t) \mathbf{Q}(t-\tau)\right\}, \quad t=1,2, \ldots, n .
$$

Finally, in a different analysis, we assumed that the population vector provides both directional and distance information about the trajectory in space. The predicted distance traveled in time bin $t$ was assumed to be equal to the length of the population vector at time $t-\tau, p(t-\tau)$, where

$$
p(t-\tau)=|\mathbf{P}(t-\tau)| .
$$

This means that the normalized trajectory based entirely upon the population vector is

$$
\left\{p(t-\tau) \mathbf{Q}(t-\tau) / p_{\max }\right\}, \quad t=1,2, \ldots, n,
$$


Figure 1. An example of population coding of movement direction. The blue lines represent the vectorial contributions of individual cells in the population $(N=475)$. The movement direction is in yellow and the direction of the population vector in red.

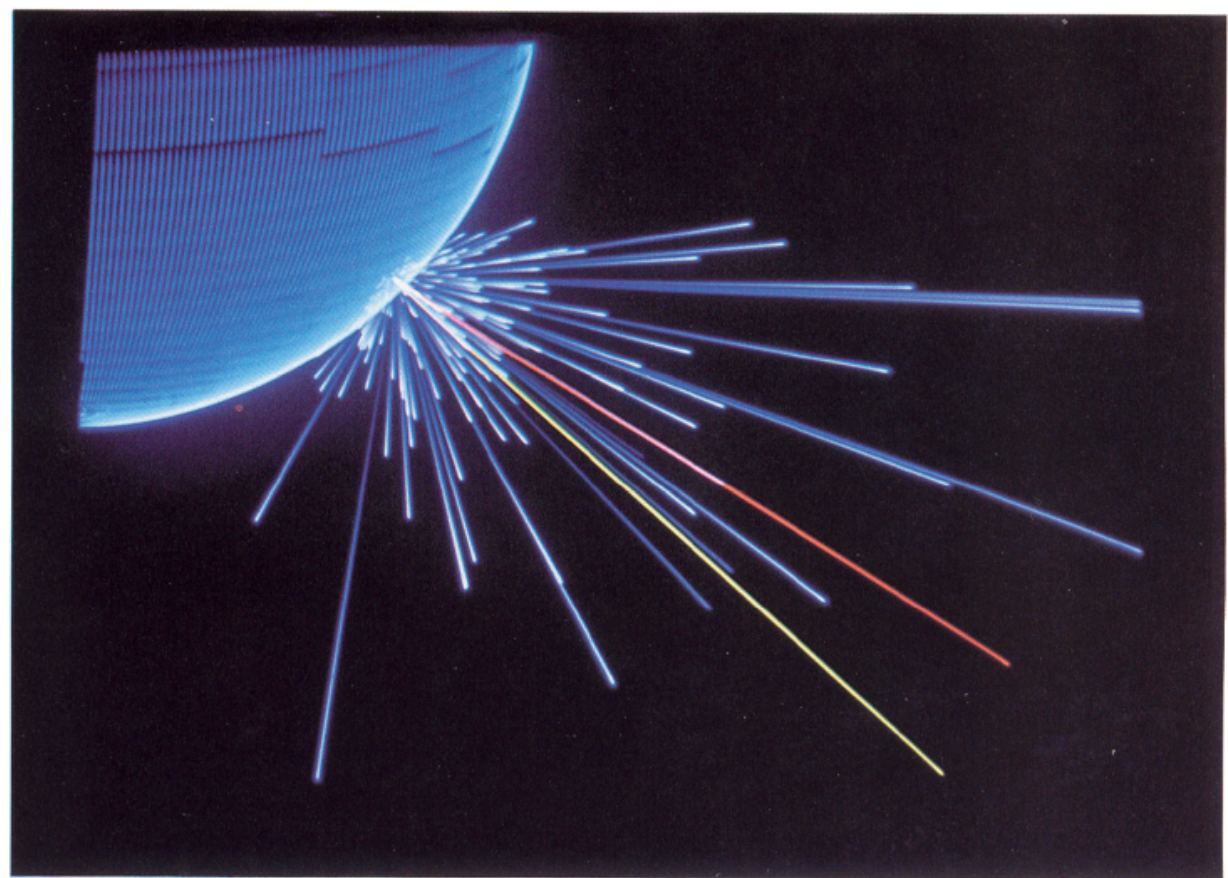

Results

$$
\left\{\mathbf{P}(t-\tau) / p_{\max }\right\}, \quad t=1,2, \ldots, n .
$$

The direction of the population vector was close to the direction of the movement. Overall, the direction of the population vector and the direction of the corresponding movement were strongly correlated ( $\rho=0.990, n=8$ directions, $p<0.001$; weighting function 8 in Appendix 2; see Table 2). An example is shown in Figure 1. The blue lines represent the vectorial contributions of the individual cells when the movement was in the direction indicated by the yellow line. The direction of the population vector is the red line. The angle between the direction of movement and the population vector was $13.7^{\circ}$. The average angle between the population and movement vector across the 8 movement directions tested was $14.6^{\circ}$. The $95 \%$ directional conmathematical analyses are described in Appendix 3.

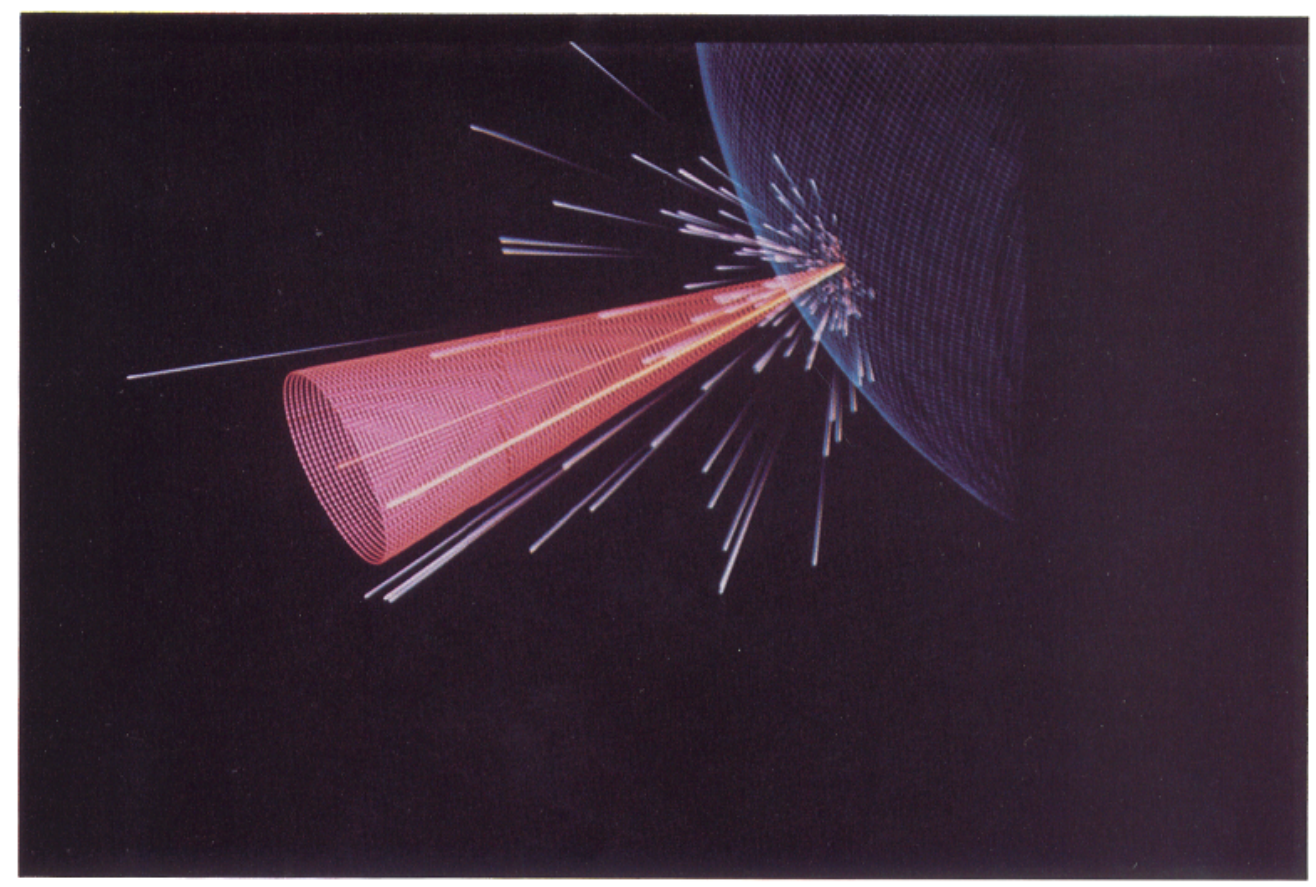

Figure 2. A $95 \%$ directional variability cone around the population vector (red). The population is the same as in Figure 1, but the movement direction (yellow) is different. 
POP. VECTOR VARIABILITY DECREASES WITH POP. SIZE

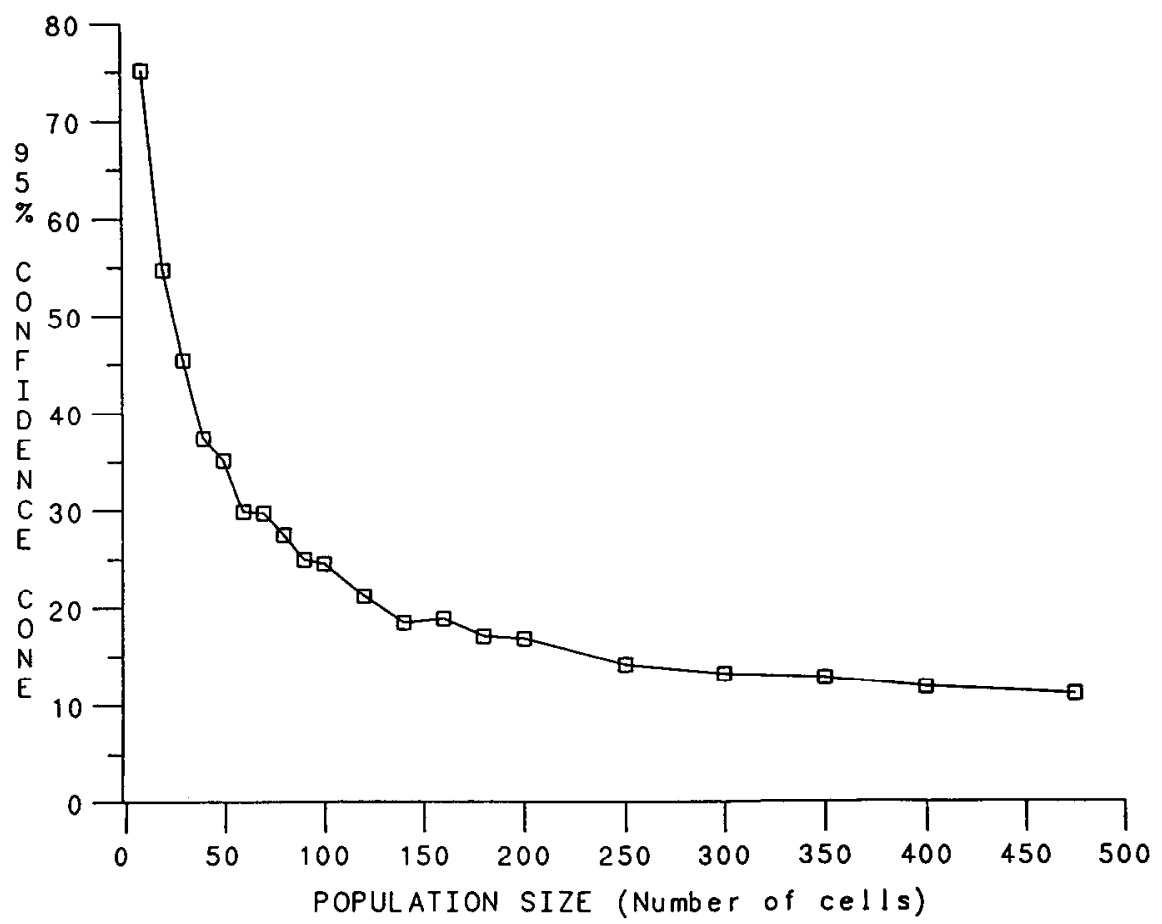

Figure 3. Directional variability of the population vector (ordinate) plotted against population size (abscissa). Points in the graph are means of 8 half-angles of $95 \%$ variability cones, 1 for each of the 8 movement directions used. Units in ordinate are in degrees. fidence cone is shown in Figure 2; the half-angle $\delta$ at the apex of the cone was $11.2^{\circ}$. The average $\delta$ was $10.6^{\circ}$ for the 8 movements. The values of these $\delta$ 's were obtained using both random sampling of the population and incorporating the trial-to-trial variability in cell discharge (see Materials and Methods). The average $\delta$ when only random sampling of the population was used was $6.2^{\circ}$; when only the trial-to-trial variability was introduced in the discharge rate of the same cells in the original population, the average $\delta$ was $8.4^{\circ}$.

The variability in the direction of the population vector decreased as the population size increased. This is shown in Figure 3 , in which the mean of $8 \delta$ 's (one for each movement direction) is plotted against the population size. It can be seen that the curve tends to an asymptote after a population size of approximately $N=150$ cells. At $N=475$, the average $\delta$ was $11.2^{\circ}$.

The results described above were based on the calculation of the population vector using weighting function 8 (see Appendix 2). Various other measures, observed experimentally or predicted from the tuning Equation 11 (see Appendix 1), also resulted in population vectors that were significantly correlated with the direction of movement. These results are shown in Table 2 (see Appendix 2).

\section{Evolution in time of the population vector}

A different, and crucial, question is whether the population vector calculated from the actual, ongoing time-varying discharge of cells during the reaction time, i.e., before the onset of movement, can predict the direction of the upcoming movement. Indeed, this was the case. An example is shown in Figure 4, in which the target direction, the time series of the population vector, and the instantaneous velocity of the movement are plotted as projections onto the frontal and sagittal planes. It can be seen that the direction of the population vector and of the movement are close to the target direction. It can be seen in
Figure 4 that the population vector points in the direction of movement $160 \mathrm{msec}$ before the onset of movement. It is important to emphasize that these results were obtained using the actually observed, not predicted, discharge rates and that a fine time grain of successive, nonsliding, nonsmoothed time windows of $20 \mathrm{msec}$ was used. This demonstrates that the population code proposed holds well for experimentally observed neural data and a fine time resolution.

\section{Neural representation of the upcoming movement trajectory}

Figure 5 displays superimposed actual (yellow) and neural rep-

Table 2. Spherical correlations ( $\rho$ ) and average angles $(\phi)$ between movement and population vectors calculated using various weighting functions, as defined in Appendix 2

\begin{tabular}{lll}
$\begin{array}{l}\text { Weighting } \\
\text { function }\end{array}$ & $\begin{array}{l}\rho \\
(\mathrm{deg})\end{array}$ & $\begin{array}{l}\phi \\
(\mathrm{deg})\end{array}$ \\
\hline 1 & 0.484 & 34.7 \\
2 & 0.963 & 16.1 \\
3 & 0.644 & 29.4 \\
4 & 0.978 & 13.5 \\
5 & 0.881 & 17.0 \\
6 & 0.975 & 13.1 \\
7 & 0.466 & 34.9 \\
8 & 0.990 & 14.6 \\
9 & 0.602 & 29.9 \\
10 & 0.996 & 9.8 \\
11 & 0.899 & 16.0 \\
12 & 0.994 & 11.8
\end{tabular}

The probability levels for $\rho$ were calculated using the randomized permutation method described by Fisher and Lee (1986). The significance levels obtained for all weighting functions were $p<0.005$ or lower. 


\section{Front View}

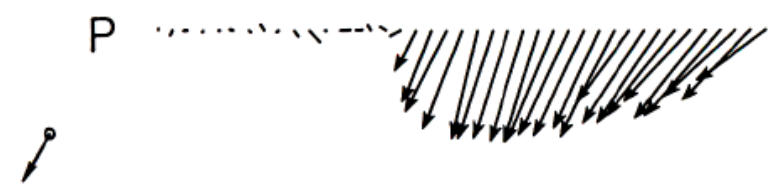

$\mathrm{M}$

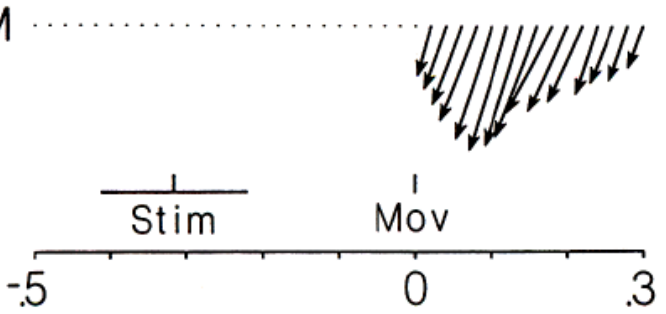

sec

\section{Side View}

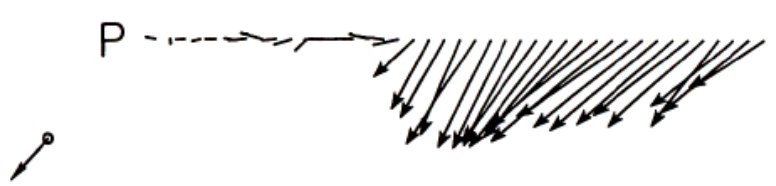

$\mathrm{M}$

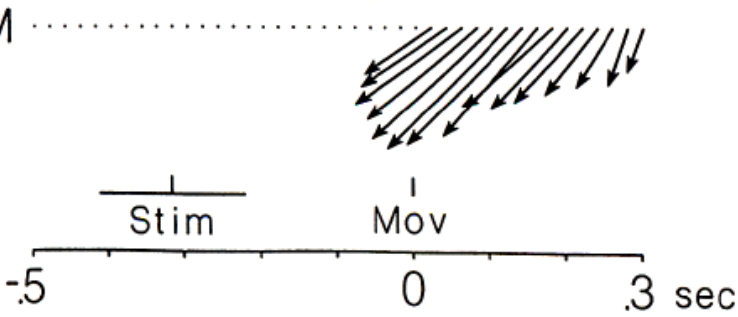

Figure 4. Evolution of the population vector in time. Front and side views of time series of population $(\mathbf{P})$ and movement $(\mathbf{M})$ vectors are shown. Population and movement vectors are normalized relative to their respective maximum. Movement vectors are averages from one animal. STIM, onset of target light; $M O V$, onset of movement.

resentations (red) of trajectories normalized and constructed as described in Materials and Methods. The "neural" trajectory was constructed based on both directional and length information obtained from the population vector. It can be seen that the spatial correspondence between the 2 kinds of trajectories is close. This suggests that the trajectory of the upcoming movement can be visualized in the motor cortex using these techniques before it unfolds in 3-D space. Very similar results were obtained when only directional information was used from the population vector.

\section{Theoretical considerations concerning the neuronal population coding}

A mathematical analysis of the operations involved in the population coding described above is given in Appendix 3. The results of this analysis showed that the following 3 basic conditions are sufficient for the population coding to predict per-

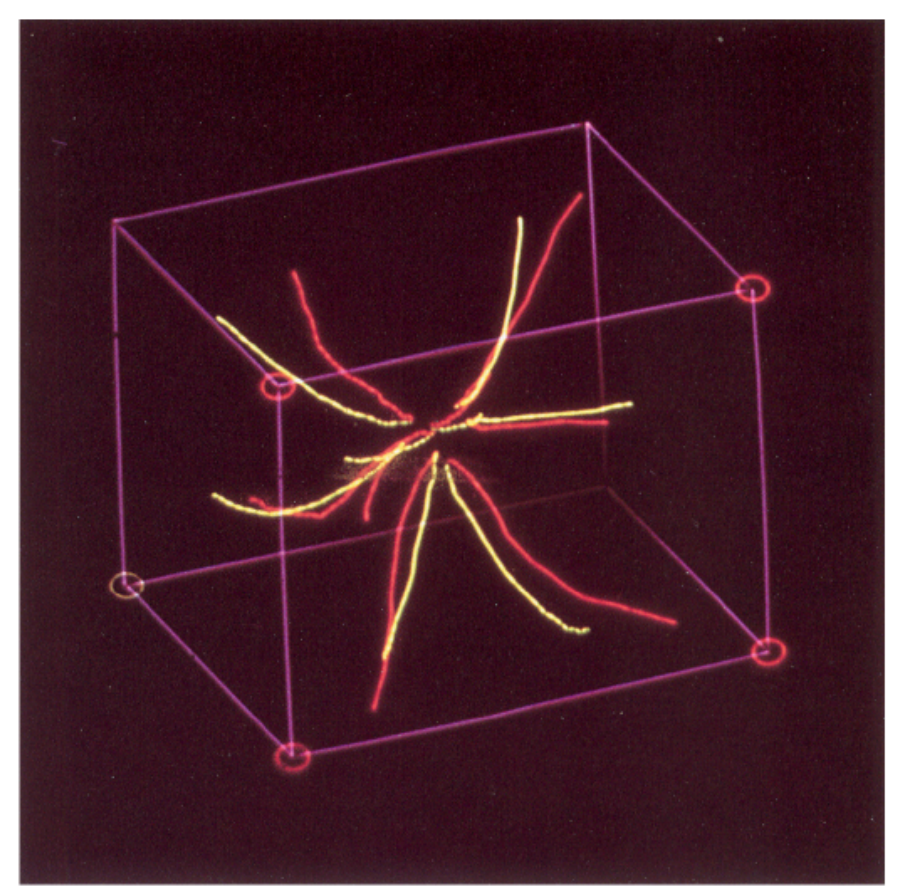

Figure 5. Normalized actual (yellow) and "neural" (orange) trajectories. See text for details.

fectly the direction of movement: first, that the directional tuning function be any of a broad category of functions that are radially symmetric around a preferred direction; second, that the preferred directions be distributed uniformly in 3-D space; and third, that the values of the tuning parameters $b$ and $k$ (see Eq. 11) be randomly distributed with respect to the preferred direction. It is reasonable to suppose that the successful coding of movement direction by the neuronal population demonstrated in the present study reflects the fact that the conditions above were fulfilled by the cells in the population. Indeed, that is the case. First, the directional tuning function described by Equation 11 (see Appendix 1) is radially symmetric around a preferred direction. Second, the preferred directions of cells in the population were distributed throughout 3-D space. This is shown in Figure 6, which depicts an equal-area projection plot of the preferred directions. (The plot was constructed as described in Schwartz et al., 1988). The preferred directions of the 475 directionally tuned cells are shown as squares or crosses, corresponding to directions in the upper or lower hemisphere, respectively, of the unit sphere. Third, the values of the tuning parameters $b$ and $k$ seem to be independent of the preferred direction; this is illustrated in Figure 7, $A$ and $B$, respectively. Each plot is the same equal-area projection plot of the preferred directions as in Figure 6, but the size of the symbols is proportional to the value of the corresponding tuning parameter. It can be seen that the magnitude of these parameters, and therefore the size of the symbols, varies throughout the distribution of the preferred directions so that there is no particular area in the distribution associated with large or small values only. These data show that the experimentally observed distributions approximate the theoretical requirements: to the extent that they are not perfect, the correspondence observed between the direction of the population vector and that of the movement will also not be perfect. 


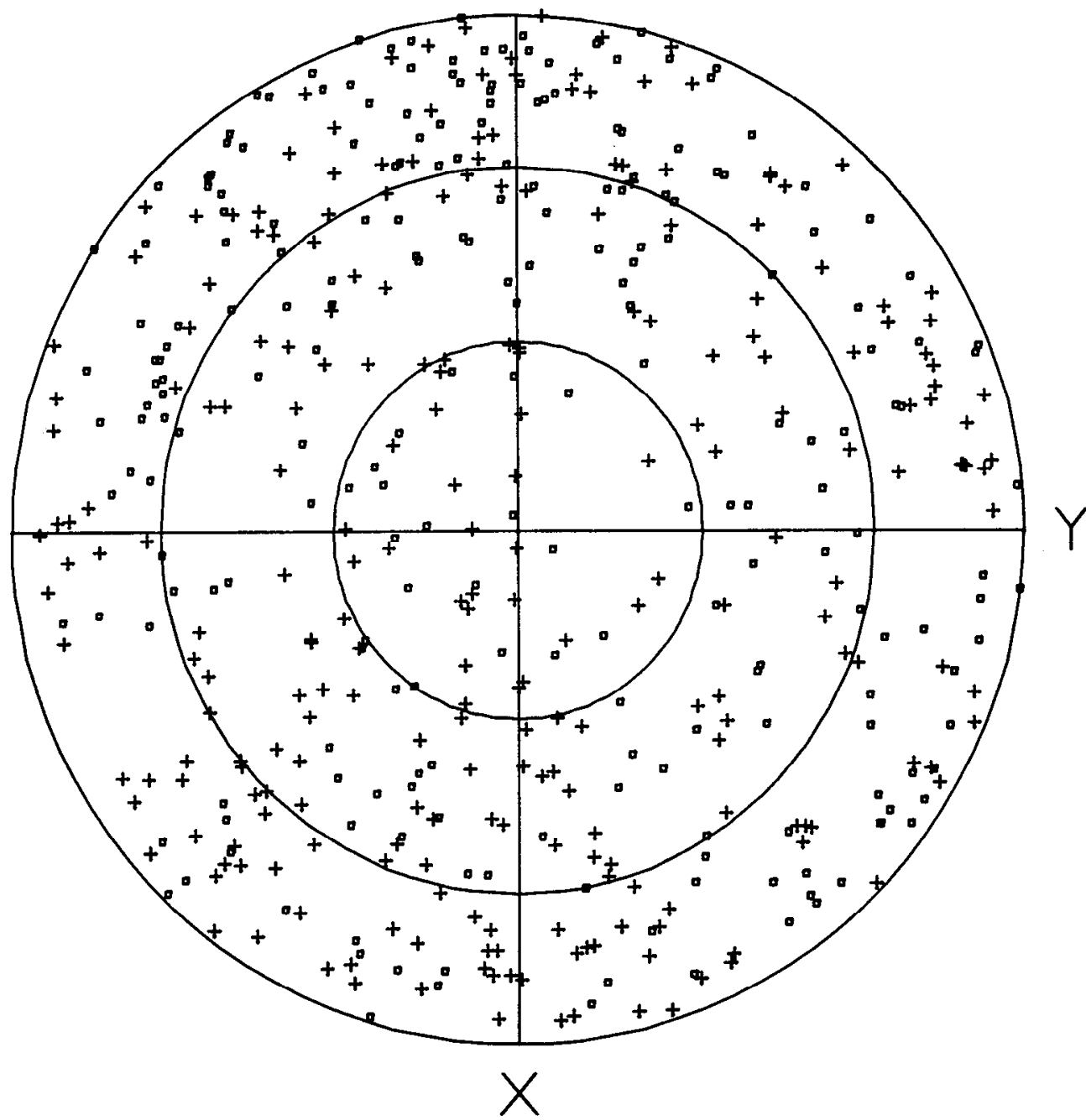

Figure 6. Equal-area projection plot of preferred directions $(N=475)$.

\section{Discussion}

Behavioral performance and neuronal populations

There is little doubt that most, if not all, functions subserved by the brain involve the combined activity of neuronal populations (Pitts and McCulloch, 1947; Mountcastle, 1967; Erickson, 1974; Johnson, 1974; Edelman and Mountcastle, 1978; Arbib, 1981). Studies of single-cell activity have shown that the presentation of a sensory stimulus, or performance in motor tasks, is associated with changes in the discharge of many ncurons in each of many brain areas. The question is how a particular function (e.g., judging the quality of a stimulus or planning and executing a movement) is realized by the corresponding neuronal ensemble(s).

An indication concerning which aspects of population activity are relevant to a certain function has been provided by careful analysis of the properties of single cells in comparison with the psychophysical capacities of human subjects or animals: Can behavioral performance be accounted for by the properties of single cells? If so, no additional principles of population action need to be invoked: in theory, at least, the behavioral capacities in question could be subserved by a neuronal population consisting of functionally homogeneous elements, that is, of cells with the same properties. For example, localization of a stimulus on the body surface, or in the visual field, is probably subserved by the activation of cells in the somatic sensory, or visual, areas, respectively, consisting of neurons with receptive fields in the part of the body, or the retina, stimulated. However, in other cases, behavioral capacities cannot be explained on the basis of the functional properties of single cells, for the relevant information may be available only at the population level. For example, the responses of quickly adapting primary skin afferents carry information concerning the frequency but not the intensity of a vibratory stimulus, although that intensity can be judged very well psychophysically (Talbot et al., 1968): this suggests that the coding of vibratory intensity is carried by the population of fibers activated. Indeed, this has been shown to be the case (Johnson, 1974). Population codes for other systems have been discussed by several investigators (Erickson, 1974; McIlwain, 1975; Kim and Molnar, 1979; Sachs and Young, 1979; Heetderks and Batruni, 1982; van Ginspergen et al., 1987).

\section{Neuronal representation of the direction of arm movement in space: neuronal population coding}

The direction of an arm movement in space is an important factor to which neuronal activity in the motor and posterior parietal (area 5) cortex relates (Georgopoulos et al., 1982; Kalaska et al., 1983, 1985; Schwartz et al., 1988). However, the frequency of discharge of single neurons rarely changes in association with only one direction of movement. Instead, single 

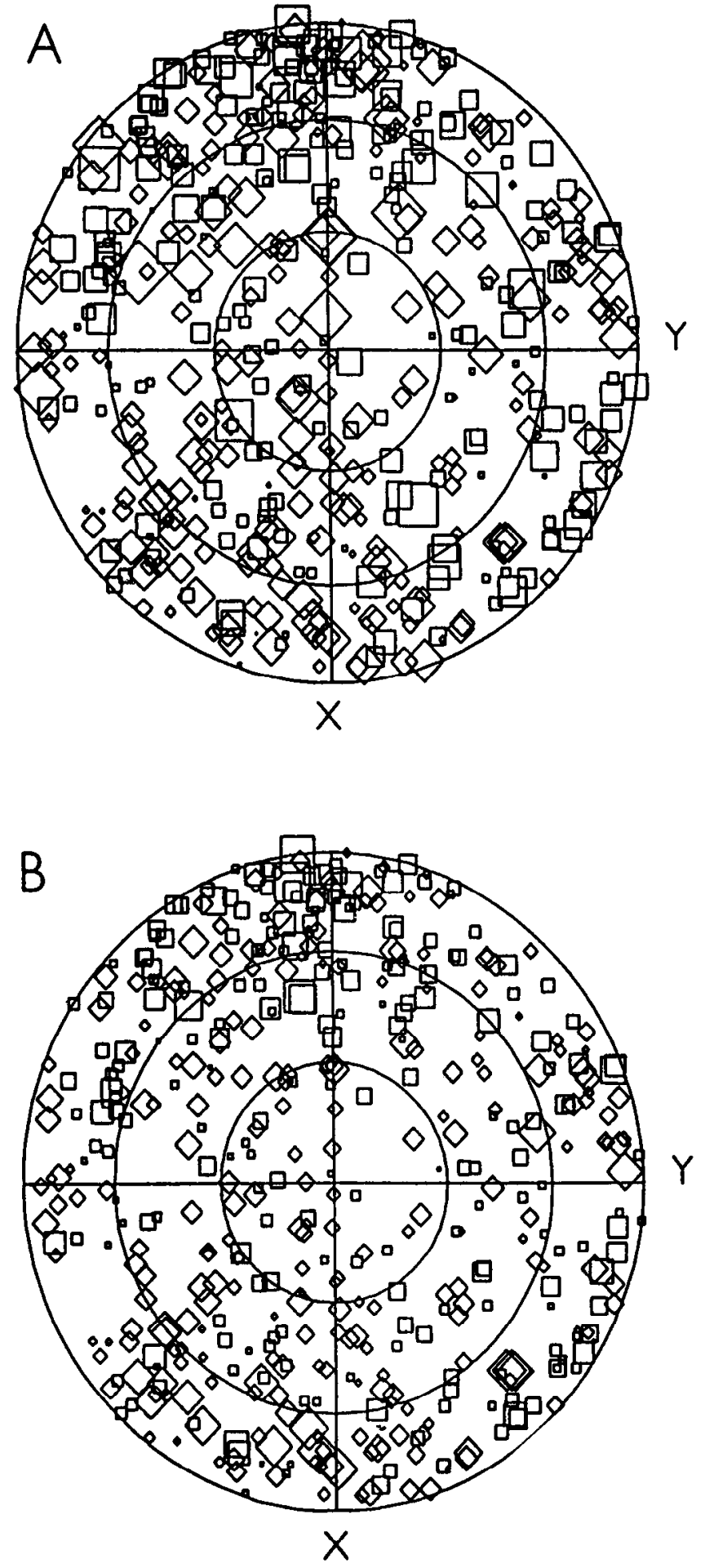

Figure 7. Equal-area projection plots of tuning parameters versus preferred direction. The size of the symbols is proportional to the value of the parameters $b(A)$ and $k(B)$.

cells discharge with movements in many directions but at different rates. The result is a directional tuning function that is broad: it possesses a peak in the cell's preferred direction but also an appreciable spread that extends throughout the directional continuum. This means that many cclls in the cnsemble will be active with movements in any particular direction and suggests that the neuronal ensemble itself is the relevant "neural unit" that carries unique advance information concerning the direction of the upcoming movement. The analyses presented in this paper concern a population code that can provide that information.

The essence of the code proposed previously (Georgopoulos et al., 1983) and extended in the present studies is that individual cells make weighted vectorial contributions ("vote") along the axis of their preferred direction. It was found that the vector sum of these contributions, that is, the population vector, points in the direction of the movement. The results of Appendix 2 (see Table 2) indicate that the code is robust with respect to the weight chosen, although normalized weights resulted in more accurate predictions. They also show that the population vector predicted well the direction of movement when using actually observed discharge rates (weighting functions 1-6 in Appendix 2) or rates predicted from the tuning equation 11 (weighting functions 7-12 in Appendix 2); in fact, the accuracy of the prediction of the movement direction by the population vector depended more on the kind of weighting function itself rather than whether it was based on actually observed or predicted rates. These considerations are important for they have different implications: the fact that the population code works for the experimentally observed data indicates that these actual data are sufficient, that is, that there is no need for more regular, smoother data for accurate predictions to be obtained; however, the fact that accurate results were also obtained using discharge rates predicted from Equation 1 is of more general significance, for it allows application of the population coding scheme to movement directions other than those studied in the present experiments.

\section{Directional tuning, population coding, and muscle events}

The population coding described in this paper can best be viewed as a general case of coding directional variables for it has been applied successfully to other cases that do not deal with the control of muscles; for example, the population vector constructed from broadly tuned directional responses of visual neurons in parietal area 7 predicted well the direction of a moving visual stimulus (Steinmetz et al., 1987). However, the present application to motor cortex can provide an insight into the motor cortical control of movement.

Recent studies (Fetz and Cheney, 1980; Shinoda et al., 1981; Lemon et al., 1986; see also Schwartz et al., 1988, for discussion) have shown that single motor cortical cells commonly engage several motoneuronal pools than just one and that, within a particular pool that is engaged, the connections of the corticomotoneuronal cell are widespread (Lemon and Mantel, 1987). This "one-to-several" relation between motor cortical cells and motoneuronal pools, and the reasonable assumption that a particular motor cortical cell could engage different pools at different strengths, may explain the observation of the present study that the preferred directions of single motor cortical cells differ among different cells and are distributed throughout 3-D space (Fig. 6; see also Schwartz et al., 1988). Thus, the multiplicity of preferred directions is in accordance with the suggestion that single cells may relate to groups of muscles, so that a particular cell may engage several motoneuronal pools in a weighted fashion: the relative ratios of those weights would then determine the preferred direction of the muscle group involved, that is, the direction of movcment with which that muscle group is most active. Given that there can be many combinations of 
engaging different motoneuronal pools with different weights, the number of the resulting preferred directions can be very large. Therefore, what seems to be represented in the motor cortex is a combinatorial aspect of motoneuronal engagement.

According to this viewpoint, the broadness of the directional tuning of single cells has certain implications: it means that the muscle group to which the cell relates will be engaged in many directions of movement, although at different intensities of activation. Thus, the resultant movement of the arm is regarded in this scheme as the outcome of coactivation of many muscle groups, each of which is being controlled as a separate functional unit. Then, the motor cortical population reflects the totality of this operation, the spatial outcome of which is movement in the desired direction. The population coding scheme proposed in this paper provides a way by which this operation at the neuronal population level can be described and visualized.

The proper matching of the output of motor cortical populations to spinal motor mechanisms with respect to reaching in space is a product of evolution and of the long period of trialand-error during which infants learn to reach accurately in space (see Georgopoulos, 1986, for a review). Recent advances in our understanding of the spinal neuronal systems subserving reaching (Lundberg, 1979; Alstermark et al., 1986a, b) offer indications as to how motor cortical control of reaching could be exerted at the spinal level. This subject has been discussed in the first paper of this series (Schwartz et al., 1988).

\section{Theoretical aspects of the population code for movement direction}

Appendix 3 shows analytically that a large class of weighting functions will yicld perfect predictions provided that the weighting functions are radially symmetric around the preferred direction, that the preferred directions are uniformly distributed in space, and that the tuning parameters are independent of preferred direction. These, indeed, are theoretically sufficient conditions for the code to be perfect. The fact that the code yields results that are close to those experimentally observed indicates the proximity of the experimental distributions to the theoretically perfect ones. Therefore, the good prediction by the population vector of the direction of the movement is not just due to the large number of neurons in the ensemble but to the fact that the experimentally observed distributions are of the proper kind. The actual results can then be considered as experimental, and therefore somewhat noisy, realizations of the perfect case.

\section{Directional variability of the population vector as a function of the population size}

The directional variability of the population vector decreased as the population size increased. Conversely, Figure 3 shows that the population code is relatively immune to cell loss: only when the population size was reduced below ca. 150 cells did the directional variability of the population vector increase appreciably and rapidly. A different aspect of the curve shown in Figure 3 concerns the minimum number of cells in the population that could provide a good estimate of the direction of the upcoming movement; this number is approximately 200-300 cells. This raises the possibility that information concerning the direction of movement in space could be adequately processed by many functionally distinct neuronal ensembles rather than by a single, large population. However, we have no evidence, at present, to support one or the other of these alternatives.

\section{Evolution in time of the population vector: time processes involved in planning the direction of arm movement in space}

The generation of movement is a process that evolves in time. Given the instructions that define the task, appropriate signals trigger the series of brain events that ultimately result in generating the movement desired. The spatial goal of reaching is that the movement to be directed to the target. Therefore, we suppose that the appearance of the target initiates the neural processes that will direct a movement in the appropriate direction. The method employed in this study permits the visualization, through the population vector, of a spatial plan of the movement as it is being formed during the reaction time. This provides a fine grain for tracing the formation of the motor cortical command for the direction of movement. Indeed, a directional signal in the population vector appeared after the presentation of the target and before the movement began, and pointed in the dircetion of the movement dictatcd by the target light. In other experiments (Crutcher et al., 1985) monkeys were trained to withhold their movement towards a light and move towards it later, after $0.5-3.2 \mathrm{sec}$, in response to its dimming. It was found that the population vector lengthened approximately $100 \mathrm{msec}$ after the onset of the light and pointed in the direction of the upcoming movement during the delay period imposed. Of course, in all these cases the population events and the population vector were reconstructed post hoc; however, it is reasonable to assume that similar, and probably more accurate, results would be obtained if the cells composing the ensemble studied had been recorded simultaneously during the course of a single movement.

The population vector provides a potentially powerful tool for interpreting neural events in relation to various aspects of behavior. An example was presented in this paper when "neural" trajectories of the upcoming movement were constructed from the population vector derived for successive time intervals during the reaction time and the spatial plan of the unconstrained movement was thus visualized (Fig. 5). We predict that more complicated trajectories could also be visualized in the same way; for example, trajectories through a maze or trajectories that are planned ahead to go around an obstacle. In these cases, the "neural" trajectories constructed from the population vector before the movement starts would show the directional turns dictated by the instructions or physical constraints and implemented by the movement that follows. These predictions can be tested in appropriately designed experiments.

\section{Appendix 1: Directional tuning function}

Let $X, Y, Z$ be the positive axes of a Cartesian coordinate system with origin at the starting point of the movement (center button). The movement direction in 3-D space will be represented by a vector $\mathbf{M}$ of unit length that makes angles $\chi, \psi$, and $\omega$ with axes $X, Y, Z$, respectively. The vector $\mathbf{M}$ is specified by its $x, y, z$ components $\left(m_{x}, m_{1}, m_{z}\right.$, respectively), where

$$
m_{y}=\cos \chi, \quad m_{y}=\cos \psi, \quad m_{z}=\cos \omega
$$

and

$$
\left(m_{x}^{2}+m_{y}^{2}+m_{z}^{2}\right)^{1 / 2}=1 .
$$

We adopt the notation that vectors are capitalized and in boldface.

The discharge rate of each cell in the population during the total experimental time (TET epoch, from the onset of the target until the end of the movement) can be described by a cosine function of the direction of movement according to the following directional tuning function:

$$
d_{i}(\mathbf{M})=b_{i}+k_{i} \cos \Theta_{\mathrm{C}, \mathbf{M}}
$$


where $d_{i}(\mathbf{M})$ is the predicted discharge rate of the $i$ th neuron with movement in direction $\mathbf{M}, b_{i}$ and $k_{i}$ are constants, $\mathbf{C}_{i}$ is the neuron's preferred direction, and $\Theta_{\mathrm{C}_{i} M}$ is the angle formed between $\mathbf{C}_{i}$ and $\mathbf{M}$. The statistical methods by which $b_{i}, k_{i}$, and $\mathbf{C}_{i}$ are calculated are described in Schwartz et al. (1988). Equation 8 can potentially yield negative values for $d_{i}(\mathbf{M})$ (see Schwartz et al., 1988). In those cases, $d_{i}(\mathbf{M})$ was set to zero for the analyses in which Equation 11 was used.

\section{Appendix 2: Weighting functions}

The following weighting functions were tried. Given 8 movement directions, $n$ repetitions of each movement, and the observed discharge rate $d_{i}^{\prime}\left(\mathbf{M}_{i}, k\right)$ of the $i$ th neuron during the TET epoch of the $k$ th repetition of the $j$ th direction, we define the following for a particular movement direction $\mathbf{M}_{\text {: }}$

(a) The average observed discharge rate across repetitions of one movement direction:

$$
D_{i j}^{\prime}=1 / n \sum_{k=1}^{n} d_{i}^{\prime}\left(\mathbf{M}_{j}, k\right)
$$

(b) The grand average rate across repetitions and all movement directions:

$$
\bar{D}_{i j}=1 / 8 \sum_{j=1}^{8} D_{i j}^{\prime}
$$

(c) The half-range of $D^{\prime}$ for 8 movements:

$$
\left.R_{i j}=\text { (maximum } D_{l j}^{\prime}-\text { minimum } D_{i j}^{\prime}\right) / 2
$$

(d) The predicted discharge rate based on the tuning function:

$$
D_{i j}=d_{i}\left(\mathbf{M}_{i}\right)=b_{i}+k_{i} \cos \theta_{c, \mathrm{M}}
$$

where $\Theta_{C, M}$ is the angle between the cell's preferred direction and the direction of movement.

We used the following weighting functions (for reasons of simplicity we omit the subscripts).
1. $D^{\prime}$
2. $D^{\prime}-\bar{D}^{\prime}$
3. $D^{\prime} / R$
4. $\left(D^{\prime}-\bar{D}^{\prime}\right) / R$
5. $D^{\prime} / \bar{D}^{\prime}$
6. $\left(D^{\prime}-\bar{D}^{\prime}\right) / \bar{D}^{\prime}$
7. $D$
8. $D-b$
9. $D / k$
10. $(D-b) / k$
11. $D / b$
12. $(D-b) / b$

Weighting functions $1-6$ and 7-12 are based on observed and predicted discharge rates, respectively. Weighting functions 1 and 7 use unnormalized rates, whereas functions 2-6 and 8-12 are normalized in various ways. (Weighting function 8 was used in the analyses described in the text.) The measures $\bar{D}^{\prime}$ and $R$ are approximations of $b$ and $k$, respectively. The results obtained with these functions are summarized in Table 2 . It can be seen that all functions yielded high and statistically significant spherical correlations, although the best predictions were obtained with normalized weights.

\section{Appendix 3}

The following is a mathematical proof which states that for a class of radially symmetric weighting functions, population vectors generated by the population equation described in Materials and Methods section (Eq. 1) will predict the direction of movement if several assumptions are made.

\section{Assumptions}

1. The preferred directions, $\mathbf{C}_{\text {, }}$ are uniformly distributed on the unit sphere.

2. Each weighting function, $w_{i}$, is radially symmetric with respect to $C_{i}$ but can vary from neuron to neuron so that

$$
w_{i}(\mathbf{M})=w\left(\Theta_{\mathbf{C}_{i} \mathbf{M}} ; \beta_{i 1} \ldots \beta_{i m}\right),
$$

where $\Theta_{C_{i} \mathbf{M}}$ is the angle between $\mathbf{C}_{i}$ and $\mathbf{M}$, and $\beta_{i 1} \ldots \beta_{i m}$ is a set of $m$ parameters that specify the form of $w_{i}$ for the $i$ th neuron. Kadial symmetry results from the dependence upon the angle between $\mathbf{C}_{i}$ and $\mathbf{M}$, instead of $\mathbf{M}$ itself.

3. Each $w_{i}$ must be tuned in the sense that the average value of $w_{i}$ for movements with components in the direction of $\mathbf{C}_{i}$ is larger than for movements away from $\mathbf{C}_{i}$.

4. The distributions of $\beta_{i 1} \ldots \beta_{i m}$ and that of the $\mathbf{C}_{i}$ are independent.

5 . The population vector can be expressed as an expectation

$$
\mathbf{P}(\mathbf{M})=E\left[\mathbf{C}_{i} w_{i}(\mathbf{M})\right]
$$

where $E$ is an expected value with respect to the $\mathbf{C}_{i}$

\section{Proof}

Consider first the simpler case where the parameters $\beta_{i 1} \ldots \beta_{i m}$ do not vary. Then for the $i$ th neuron,

$$
w(\mathbf{M})=w\left(\Theta_{C_{i N}}\right)
$$

and for each weighting function,

$$
\int \mathbf{M} w\left(\Theta_{\mathbf{c}_{i} \mathbf{M}}\right) d \mathbf{M}=\delta \mathbf{C}_{i}
$$

where $\delta$ is a positive constant. The orientation of the result follows from the symmetry of $w_{i}$, which requires a resultant vector along its axis of symmetry, $\mathbf{C}_{i}$. The positivity of $\delta$ follows from Assumption 3.

Since $\mathbf{M}$ and $C_{i}$ are both directions that vary over the sphere and because $\Theta_{c, M}=\Theta_{\mathrm{M} \text { ci }}$, one can switch the roles of $\mathbf{M}$ and $\mathbf{C}_{i}$ in Equation 16 so that

$$
\int \mathbf{C}_{i} w\left(\Theta_{\mathbf{C}_{j} M}\right) d \mathbf{C}_{i}=\delta \mathbf{M}
$$

The population vector, $\mathbf{P}$, is defined by

$$
\begin{aligned}
\mathbf{P}(\mathbf{M}) & =E\left[\mathbf{C}_{i} w\left(\Theta_{(,)}\right)\right] \\
& =\int \mathbf{C}_{i} w\left(\Theta_{C, M}\right) \operatorname{prob}\left(\mathbf{C}_{i}\right) d \mathbf{C}_{i} .
\end{aligned}
$$

The uniformity of $\mathbf{C}_{i}$ implies that $\operatorname{prob}\left(\mathbf{C}_{i}\right)$ is a positive constant, which will be called $\alpha$, so that

and substituting from Equation 17

$$
=\alpha \int \mathbf{C}_{i} w\left(\Theta_{C, M}\right) d \mathbf{C}_{i}
$$

$$
=\alpha \delta \mathbf{M} \text {. }
$$

Thus, when $\beta_{i 1} \ldots \beta_{i m}$ do not vary:

$$
\mathbf{P}(\mathbf{M})=E\left[\mathbf{C}_{i} w\left(\Theta_{\mathbf{C}_{i} \mathbf{M}}\right)\right]=\alpha_{\delta} \mathbf{M} .
$$

This implies that the population vector points in the same direction as $\mathbf{M}$.

We now turn to the more general case where the parameters $\beta_{i 1} \ldots$ $\beta_{m, \prime}$ are allowed to vary. In this case,

$$
w_{i}(\mathbf{M})=w\left(\Theta_{\mathbf{M C}} ; \beta_{i 1} \ldots \beta_{i m}\right)
$$

and

$$
\mathbf{P}(\mathbf{M})=E\left[\mathbf{C}_{i} w\left(\Theta_{\mathrm{MC}} ; \beta_{i 1} \ldots \beta_{i m}\right)\right] .
$$

We can rewrite this expression because the parameters $\beta_{i 1} \ldots \beta_{i m}$ are independent of $\mathbf{C}_{i}$, so that

$$
=E\left\{E\left[\mathbf{C}_{i} w\left(\Theta_{\mathrm{Mc} i} ; \beta_{i 1} \ldots \beta_{i m}\right) \mid \beta_{i 1} \ldots \beta_{i m}\right]\right\} .
$$

However, since parameters $\beta_{i l} \ldots \beta_{i m}$ are constant for the conditional expectation, we can use the same arguments that resulted in Equation 18 to show that

$$
\begin{aligned}
& E\left[\mathbf{C}_{i} w\left(\Theta_{\mathrm{Mc}} ; \beta_{i 1} \ldots \beta_{i m}\right) \mid \beta_{i 1} \ldots \beta_{i m}\right] \\
& \quad=\alpha\left(\beta_{i 1} \ldots \beta_{i m}\right) \delta\left(\beta_{i 1} \ldots \beta_{i m}\right) \mathbf{M},
\end{aligned}
$$

where $\alpha$ and $\delta$ are now positive functions of the parameters $\beta_{i 1} \ldots \beta_{i m}$. This means that

$$
\begin{aligned}
\mathbf{P}(\mathbf{M}) & =E\left\{\alpha\left(\beta_{i 1} \ldots \beta_{i m}\right) \delta\left(\beta_{i 1} \ldots \beta_{i m}\right) \mathbf{M}\right\} \\
& =\mathbf{M} E\left\{\alpha\left(\beta_{i 1} \ldots \beta_{i m}\right) \delta\left(\beta_{i 1} \ldots \beta_{i m}\right)\right\} .
\end{aligned}
$$

Here, the expectation is positive because the functions, $\alpha$ and $\delta$, are positive. This proves that the population vector points in the direction of $\mathbf{M}$ for the more general case in which the parameters $\beta_{i 1} \ldots \beta_{i m}$ can vary.

In particular, all the weighting functions in Appendix 2 allow perfect 
prediction of movement direction under the assumptions above. For example,

$$
w_{i}(\mathbf{M})=b_{i}+k_{i} \cos \left(\Theta_{C_{i}, \mathbf{M}}\right)
$$

for positive $b_{i}$ and $k_{i}$ fits the assumptions of this proof. $w_{i}(\mathbf{M})$ is radially symmetric about $\mathbf{C}_{i}$ because $\cos \left(\Theta_{C, M}\right)$ is symmetric about $\mathbf{C}_{i}$, and $w_{i}$ is tuned because it attains its maximum for movements in the direction of $\mathbf{C}_{i}$ (i.e., when $\Theta_{C, M}$ is zero).

\section{References}

Alstermark, B., T. Gorska, T. Johannisson, and A. Lundberg (1986a) Hypermetria in forelimb target-reaching after interruption of the inhibitory pathway from forelimb afferents to $\mathrm{C} 3-\mathrm{C} 4$ propriospinal neurones. Neurosci. Res. 3: 457-461.

Alstermark, B., T. Gorska, T. Johannisson, and A. Lundberg (1986b) Effects of dorsal column transection in the upper cervical segments on visually guided forelimb movements. Neurosci. Res. 3: 462-466.

Arbib, M. A. (1981) Perceptual structures and distributed motor control. In Handbook of Physiology. The Nervous System II, American Physiological Society, pp. 1449-1480, Bethesda, MD.

Crutcher, M. D., A. B. Schwartz, and A. P. Georgopoulos (1985) Representation of movement direction in primate motor cortex in the absence of immediate movement. Soc. Neurosci. Abstr. 11: 1273.

Diaconis, P., and B. Efron (1983) Computer-intensive methods in statistics. Sci. Am. 248: 116-130.

Edelman, G. M., and V. B. Mountcastle (1978) The Mindful Brain, MIT Press, Cambridge, MA

Erickson, R. P. (1974) Parallel "population" neural coding in feature extraction. In The Neurosciences: Third Study Program, F. O. Schmitt and F. G. Worden, eds., pp. 155-169, MIT Press, Cambridge, MA.

Fetz, E. E., and P. D. Cheney (1980) Postspike facilitation of forelimb muscle activity by primate corticomotoneuronal cells. J. Neurophysiol. 44: $751-772$.

Fisher, N. I., and A. J. Lee (1986) Correlation coefficients for random variables on a unit sphere or hypersphere. Biometrika 73: 159-164.

Georgopoulos, A. P. (1986) On reaching. Annu. Rev. Neurosci. 9: 147-170.

Georgopoulos, A. P., J. F. Kalaska, R. Caminiti, and J. T. Massey (1982) On the relations between the direction of two-dimensional arm movements and cell discharge in primate motor cortex. J. Neurosci. 2: 1527-1537.

Georgopoulos, A. P., R. Caminiti, J. F. Kalaska, and J. T. Massey (1983) Spatial coding of movement: A hypothesis concerning the coding of movement direction by motor cortical populations. Exp. Brain Res. (Supp.) 7: 327-336.

Georgopoulos, A. P., J. F. Kalaska, M. D. Crutcher, R. Caminiti, and J. T. Massey (1984) The representation of movement direction in the motor cortex: Single cell and population studies. In Dynamic Aspects of Neocortical Function, G. M. Edelman, W. E. Gall, and W. M. Cowan, eds., pp. 501-524, Wiley, New York.

Georgopoulos, A. P., A. B. Schwartz, and R. E. Kettner (1986) Neuronal population coding of movement direction. Science 233: 14161419.

Heetderks, W. J., and R. Batruni (1982) Multivariate statistical analysis of the response of the cockroach giant interneuron system to wind puffs. Biol. Cyber. 43: 1-11.

Johnson, K. O. (1974) Reconstruction of population response to a vibratory stimulus in quickly adapting mechanoreceptive afferent fiber population innervating glabrous skin of the monkey. J. Neurophysiol. 37: 48-72.

Kalaska, J. F., R. Caminiti, and A. P. Georgopoulos (1983) Cortical mechanisms related to the direction of two-dimensional arm movements: Relations in parietal area 5 and comparison with motor cortex. Exp. Brain Res. 51: 247-260.

Kalaska, J. F., D. A. D. Cohen, and M. C. Hyde (1985) Differences in the spatial relation between movement direction dependent and load direction-dependent activity changes in primate cortex areas 4 and 5. Soc. Neurosci. Abstr. 11: 1273.

Kim, D. O., and C. E. Molnar (1979) A population study of cochlear nerve fibers: Comparison of spatial distributions of average-rate and phase-locking measures of responses to single tones. J. Neurophysiol. 42: $16-30$.

Lemon, R. N., and G. W. H. Mantel (1987) Direct facilitation by corticospinal neurons of single motor units in the hand muscles of the conscious monkey. Soc. Neurosci. Abstr. 13: 243.

Lemon, R. N., G. W. H. Mantel, and R. B. Muir (1986) Corticospinal facilitation of hand muscles during voluntary movement in the conscious monkey. J. Physiol. (Lond.) 381: 497-527.

Lundberg, A. (1979) Integration in a propriospinal motor centre controlling the forelimb in the cat. In Integration in the Nervous System, H. Asanuma and V. S. Wilson, eds., pp. 47-69, Igaku-Shoin, Tokyo.

McIlwain, J. T. (1975) Visual receptive fields and their images in superior colliculus of the cat. J. Neurophysiol. 38: 219-230.

Mountcastle, V. B. (1967) The problem of sensing and the neural coding of sensory events. In The Neurosciences: An Intensive Study Program, F. O. Schmitt, G. Quarton, and T. Melnuchuk, eds., pp. 393-408, Rockefeller Univ. Press, New York.

Pitts, W., and W. S. McCulloch (1947) How we know universals. The perception of auditory and visual forms. Bull. Math. Biophys. 9:127147.

Sachs, M. B., and E. D. Young (1979) Encoding of steady-state vowels in the auditory nerve: Representation in terms of discharge rate. $\mathrm{J}$. Acoust. Soc. Am. 66: 470-479.

Schwartz, A. B., R. E. Kettner, and A. P. Georgopoulos (1986) Population coding of 3-dimensional movement direction in primate motor cortex: Confidence intervals, robustness, and evolution in time of the population vector. Soc. Neurosci. Abstr. 12: 256.

Schwartz, A. B., R. E. Kettner, and A. P. Georgopoulos (1988) Primate motor cortex and free arm movements to visual targets in threedimensional space. I. Relations between single cell discharge and direction of movement. J. Neurosci. 8: 2913-2927.

Shinoda, Y., J. I. Yokota, and T. Futami (1981) Divergent projections of individual corticospinal axons to motoneurons of multiple muscles in the monkey. Neurosci. Lett. 23: 7-12.

Steinmetz, M. A., B. C. Motter, C. J. Duffy, and V. B. Mountcastle (1987) Functional properties of parietal visual neurons: Radial organization of directionalities within the visual field. J. Neurosci. 7: 177-191.

Talbot, W. H., I. Darian-Smith, H. H. Kornhuber, and V. B. Mountcastle (1968) The sense of flutter-vibration: Comparison of the human capacity with response patterns of mechanoreceptive afferents from the monkey hand. J. Neurophysiol. 31: 301-334.

van Ginspergen, J. A. M., A. J. van Opstal, and A. A. M. Tax (1987) Collicular ensemble coding of saccades based on vector summation. Neuroscience 21: 541-555. 